\title{
Validation of microarray image analysis accuracy
}

\author{
Bruz Marzolf and Michael H. Johnson \\ The Institute for Systems Biology, Seattle, WA, USA
}

BioTechniques 36:304-308 (February 2004)

DNA microarray technology has emerged as a massively parallel and widely accepted system of gene expression analysis. In response, many microarray facilities have developed systems to handle this technology in a high-throughput manner. As these facilities evolve into production environments, it is increasingly important to establish automated statistically based quality assurance checks. Here we describe a method for quality control during the image analysis step of the microarray process.

Close examination of array image patterns reveal imperfect spacing between features. In large part, these misspacings are due to the mechanical imperfections of spotted microarray synthesizers. Typically, spotting on arrays is done with a print head containing multiple pins, each printing a grid of many features. Microarray spotters have precise motion control, often within $2 \mu \mathrm{m}$ or less. Comparatively, the feature-to-feature spacing is on the $100-\mu \mathrm{m}$ scale, keeping the features within a given grid close to ideal geometry. Grid-to-grid spacing, however, varies on the order of tens of microns (Figure 1), which leads to grid-to-grid misalignments.

Image analysis software handles this challenge with an initial grid identification step, followed by locating the features within each grid. Early versions of microarray image analysis software require a significant amount of time be spent on manual identification and alignment of grids onto array features. Many current software packages now offer automatic image processing and spotfinding. Though useful, such software is not entirely accurate and can introduce errors via misalignments for spotted arrays. A common example of such error is the misplacement of grids by full row or column offsets (Figure 2).

Misplacement of a grid causes incorrect coordinates to be assigned to each feature in that grid. Since annotations are tracked by grid coordinates, incorrect annotations are therefore assigned to each feature. Since incorrect grid placement can cause such large-scale errors, it is still necessary to perform subsequent visual inspections and manual corrections of grid placement on each microarray image. This involves looking at each grid on a spotfound image to ensure that errors such as the one demonstrated in Figure 2 are not present, which reduces the timesaving benefits of automated image analysis software $(1,2)$. Other array layout errors are produced by rotation of the print head or slide on the printing platform and movement of slides on the platform with respect to the image axes. While notable, these issues are better addressed in the spotfinding software, and here we focus on slides with negligible defects of these types.

Human, yeast, and mouse arrays were used to design and optimize the method presented here. Spotting for all arrays was performed with a VersArray Chipwriter Pro ${ }^{\mathrm{TM}}$ (Bio-Rad Laboratories, Hercules, CA, USA) using SMP3 spotting pins (TeleChem International, Sunnyvale, CA, USA) onto Corning

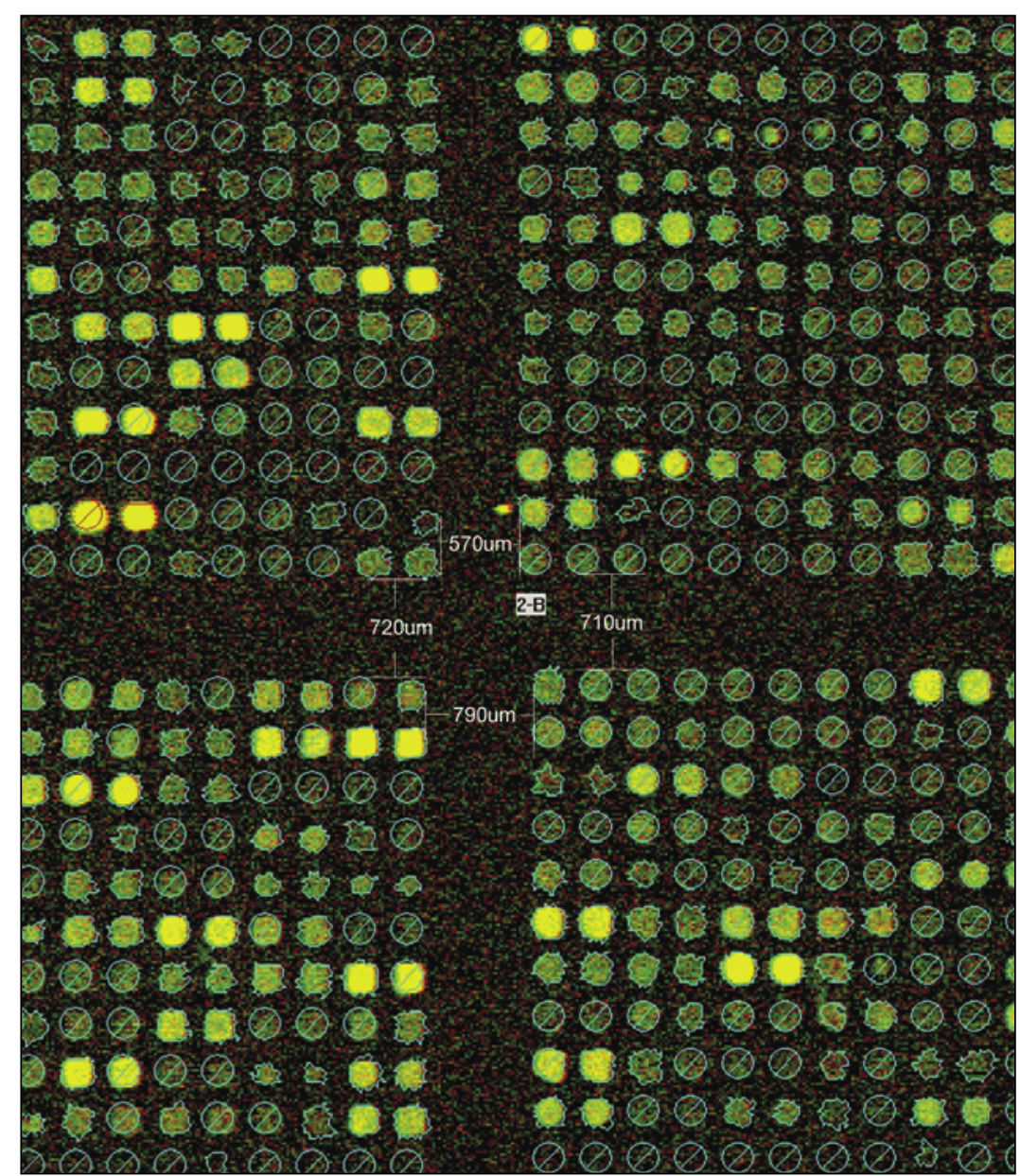

Figure 1. Grid-to-grid spacing. Imperfections in grid-to-grid spacing are large enough to be visible by eye and make spotfinding more difficult. If ideal geometry were observed, the distances between all of these grids would be equal. (Spotfound image taken from AnalyzerDG.) 
GAPS2 ${ }^{\mathrm{TM}}$ substrates (Corning, Corning, NY, USA). Human slides consisted of PCR products from the 40,032 human clones available from ResGen ${ }^{\mathrm{TM}}$ / Invitrogen (Carlsbad, CA, USA). Each PCR product was spotted a single time on each array, which was organized in 48 grids of 841 features per grid. Yeast arrays consisted of the Operon ${ }^{\circledR} 70$ mer set (Qiagen, Valencia, CA, USA) covering 6307 yeast genes. Each oligonucleotide was printed in adjacent duplicates in a supergrid of 24 grids, each containing 552 features. The supergrid itself was also printed in duplicate on the slide, for a total of 4 features per gene per slide. Mouse arrays consisted of the 16,463 oligonucleotide probes available from Qiagen as the Operon mouse genome set version 2.0. Each oligonucleotide was single-spotted on a supergrid of 24 grids, each containing 756 features, and the supergrid was duplicated as with the yeast array, yielding two features per probe. All arrays described were hybridized with cDNA labeled by synthesis in the presence of Cy ${ }^{\mathrm{TM}} 3$ - or Cy5-labeled nucleotides.

Scanning was performed with a Packard BioChip ScanArray ${ }^{\circledR} 5000$ (PerkinElmer Life and Analytical Sciences, Boston, MA, USA). All scans were acquired at $10-\mu \mathrm{m}$ pixel resolution and 16-bit range. Laser voltage was kept at $95 \mathrm{~V}$, and the photomultiplier tube (PMT) was adjusted to control intensity levels. Three software packages were used to ensure applicability across platforms where appropriate. QuantArray ${ }^{\circledR}$ (PerkinElmer Life and Analytical Sciences) and Dapple (3) are two examples of manual spotfinders, whereas AnalyzerDG ${ }^{\mathrm{TM}}$ (MolecularWare, Cambridge, MA, USA) allows automatic processing of batch sets. When unspecified, spot intensity quantitation files were generated by AnalyzerDG. Spot intensity was calculated as the mean pixel intensity inside each feature boundary. Spot background intensity

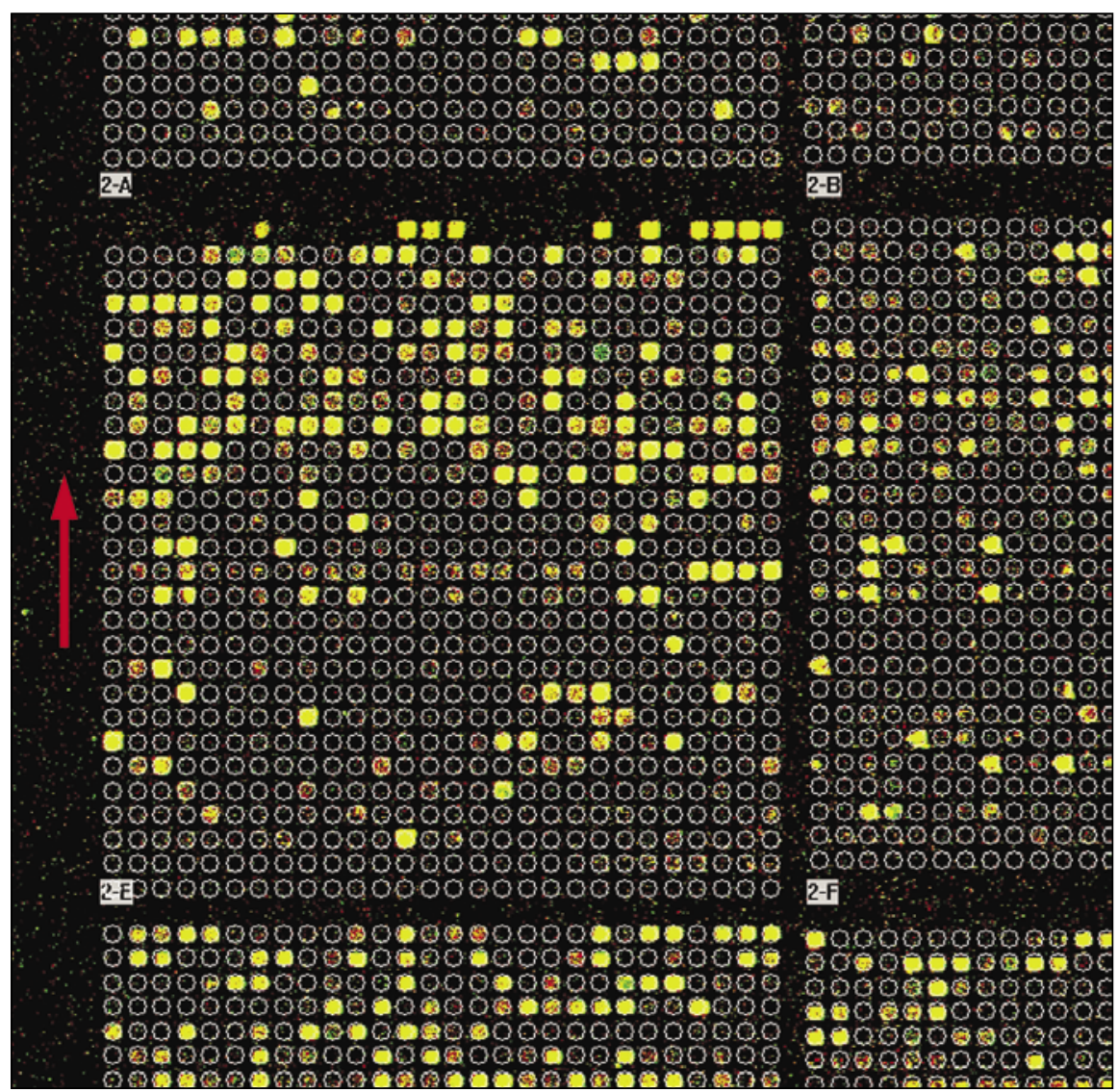

Figure 2. Misaligned grid. A typical spotfinding error, demonstrating grid placement that is one full row lower than the correct position. This type of error must be visually identified by the person doing image analysis and manually corrected. (Spotfound image taken from AnalyzerDG.) was calculated as the mean of the pixel intensities from a square cell centered on each feature with sides twice the diameter of the feature, excluding surrounding features.

Comparing hybridized array images among slides with identical feature layout, it is has been observed that there are similar patterns of feature intensities (4). We investigated this relationship, since one concern with relying upon these intensity patterns is that labeled samples from differing sources will not correlate sufficiently. We have found that even biological samples from widely different sources and preparations yield array intensities that correlate well with each other (Figure 3A). In practice, we recommend that comparisons be done among a batch of slides within one scientific project, where the hybridized samples are from very similar sources and have reliably high correlation, such as those in Figure 3. Taking advantage of these patterns, Pearson product-moment correlation coefficient $(R)$ (http:// mathworld.wolfram.com/Correlation Coefficient.html) of intensities between arrays based on location coordinates can be used to determine if spotfinding was done correctly:

$$
R=\frac{\left(\sum x y-n \overline{x y}\right)}{\sqrt{\left(\sum x^{2}-n \bar{x}^{2}\right)\left(\sum y^{2}-n \bar{y}^{2}\right)}}
$$

Since the spotfinding errors we describe affect entire grids, $R$ is checked at a grid-by-grid level and is observed to be significantly higher than zero for correct alignment and very close to zero for a misalignment (Figure 3B). In addition to determining correlation for each grid at its given location, a number of shifted positions are also assessed. If the correlation for the given grid location is not as high as another possible fit, a suggestion is made for moving the grid into proper alignment. We have developed this approach into a method for verification of the output from microarray image analysis software.

In this method, the grid placement for one slide is visually inspected and verified among a group of slides hybridized with similar biological material. The slide chosen for this purpose is typically the brightest array among the set to be compared, since correct 
placement of grids is most easily determined on slides with high intensities. This slide serves as the reference pattern to which all other slides of identical reporter content and feature layout are compared. Each grid between the reference slide and the query slide is compared based upon the sum of all channels' intensities for each feature. $R$-values are calculated for the locations specified by the image analysis software, but also for hypothetical locations resulting from shifting each grid on the test slide one or more rows or columns from its given location. $R$-values are calculated only for the intersecting region of the original and shifted grids. We allow $R$ to be assessed at any combination of rows and columns shifted, up to one-eighth of the total number of rows or columns in the grid, respectively. Shifting is limited, since incorrect grid placements are typically only a single row or column off, and assessing drastic shifts reduces the subset of spots being compared so much that spurious results can occur. Assessing the shifted locations creates a table of $R$-values corresponding to each possible shift of each grid. If the shifted location of the grid produces a significantly higher correlation coefficient than the original location, a suggestion is made on how to place the grid for best fit.

Since it is possible that the best fit is not the correct fit, it is necessary to determine criteria for a correct fit. Absence of a correct fit can occur if feature boundary detection fails or if the intensity patterns are vastly different between arrays being compared. The criterion for a correct fit is determined by calculating one-quarter of the average of the difference between the best and second best correlation for all grids. For each grid, if $R$ difference is above this cutoff, the best fit for that grid is recommended as the correct fit. If the $R$ difference is under the cutoff, it is called a "bad grid." This simply means that $R$-values were not high enough to find a correct fit for a particular grid and that it should be inspected visually. Causes for this include very low hybridization intensity and background artifacts on the slide.

The method has been implemented in PERL and is run via a web interface called GrAPE (Grid Alignment Processing Environment). GrAPE allows the user to select both a slide to use as the reference and which slides are to be tested. GrAPE then performs the correlation checks and reports the correctness of fit as HTML tables representing the grids of the arrays being compared. Users can then leave their browser open and make appropriate changes in their image analysis software. Image analysis quantitation files are exported, and a final recheck is done to ensure correct alignment on all grids after corrections. Alternatively, users can do spotfinding to the 


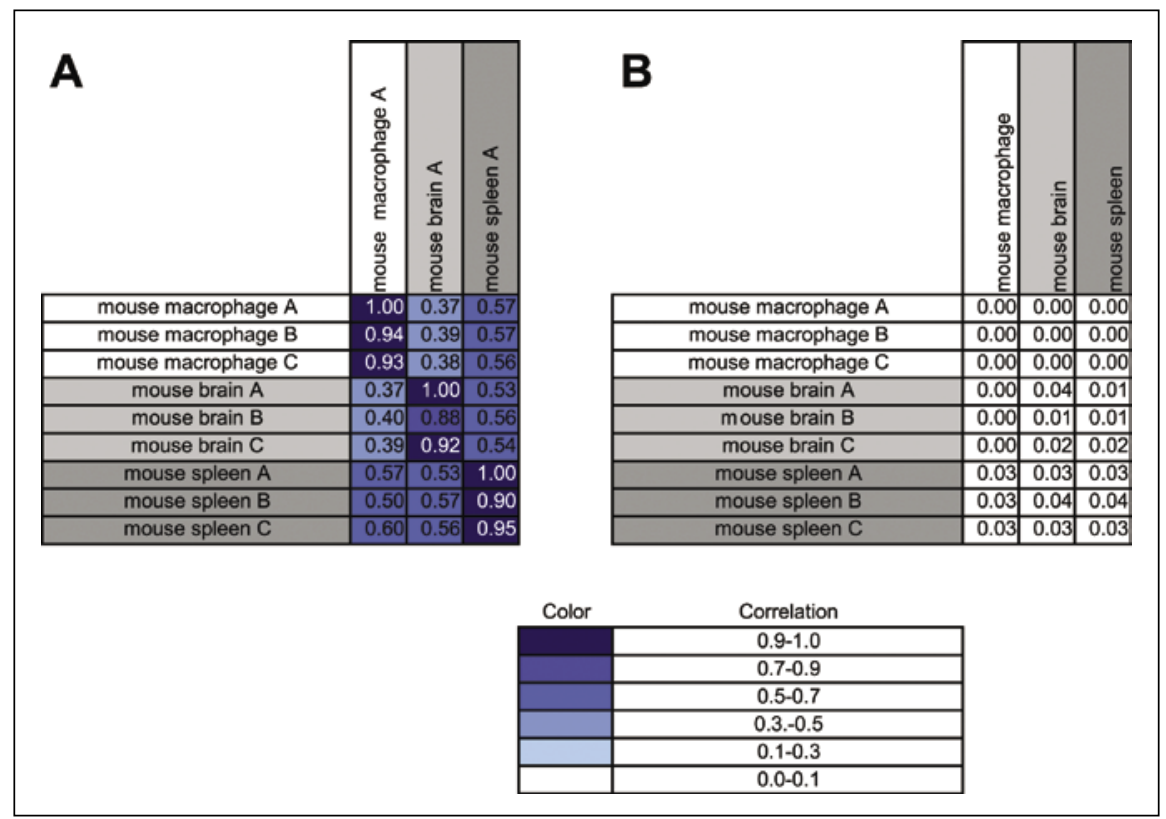

Figure 3. Correlation of various samples. (A) Summary of correlation coefficients comparing arrays hybridized with samples within and between different biological sources. (B) All of the same comparisons as seen in panel A, except that the grids were incorrectly placed by one row. These show essentially no correlation. All comparisons in this figure were performed with mouse arrays.

best of their ability by eye and then use GrAPE as a final verification of correctness. A demonstration is available at http://db.systemsbiology.net/ software/GrAPE, with a set of sample slides chosen to show the various possible outputs. The source code for this implementation is also available on the site and can be customized for other spot-intensity quantitation file formats and locations.

A variation on our method can also be used to check for printing mistakes between batches of slides that should have identical layouts. In this case, $R$ can be calculated on a plate-by-plate basis to check for missing or incorrectly oriented plates during a print.

One important concern that must be addressed is the error rate for this method. The first type of error is a false negative result, in which a suggestion is made for moving a grid that is actually correct. This is observed rarely when overall correlation between reference and query slide is high, but occurs more frequently as correlation drops. For this reason, our software issues a warning message when $R$ is below 0.7. Although this type of error is still occasionally observed when $R$ exceeds 0.7 , we feel it is not problematic since the suggestion of an incorrect grid only prompts the user to look at the image analysis, at which point they can evaluate the software's suggestion themselves. A false positive result, however, is much more problematic. If a grid is indicated as correct by the software, but is in fact wrong, it will most likely go uncorrected since the user has a false sense of security about their grid alignments. We addressed this concern by purposely introducing over 250 individual incorrect grid placements on mouse, human, and yeast arrays and found that in every case the incorrect grid was correctly identified, establishing that false positives are highly unlikely. These observations seem reasonable, since the probability of a false negative is determined by how likely one of many incorrect positions will, by chance, give a correlation higher than the original correct alignment. On the other hand, a false positive only occurs when correlation for the one incorrectly placed grid location is higher than the one shifted location that would provide correct alignment. For these reasons, we feel that the method can be used with confidence to validate grid alignment.

\section{REFERENCES}

1.Jain, A.N., T.A. Tokuyasu, A.M. Snijders, R. Segraves, D.G. Albertson, and D. Pinkel. 2002. Fully automatic quantification of microarray image data. Genome Res. 12:325-332.

2.Steinfath, M., W. Wruck, H. Seidel, H. Lehrach, U. Radelof, and J. O'Brien. 2001. Automated image analysis for array hybridization experiments. Bioinformatics 17:634-641.

3.Buhler, J., T. Ideker, and D. Haynor. 2000. DAPPLE: improved techniques for finding spots on DNA microarrays. Technical Report UWTR 2000-08-05, Department of Computer Science and Engineering, University of Washington, Seattle.

4.Reid, R., D.J. Dix, D. Miller, and S.A. Krawetz. 2001. Recovering filter-based microarray data for pathways analysis using a multipoint alignment strategy. BioTechniques 30:762-766.

Received 11 September 2003; accepted 6 November 2003.

Address correspondence to Bruz Marzolf, The Institute for Systems Biology, 1441 $N$ 34th Street, Seattle, WA 98103, USA. e-mail: bmarzolf@systemsbiology.org 